\title{
On Multicarrier Schemes For Doubly-Selective Channels
}

\author{
Aldo Cero, Alan Barbieri, Giulio Colavolpe \\ Università di Parma \\ I-43100 Parma, ITALY \\ E-Mail: cero@tlc.unipr.it, barbieri@tlc.unipr.it, giulio@unipr.it
}

\author{
Marilynn P. Wylie-Green \\ Nokia Siemens Networks \\ Irving, TX 75039 USA \\ E-Mail: marilynn.green@nsn.com
}

\begin{abstract}
We compare four different multi-carrier schemes in a discrete-time, oversampled domain over doubly-selective channels. We find that all the schemes can be implemented in a reduced complexity way, resorting to suitable fast transforms like DFT, DCT, or DST. For all models we assume either a rectangular base pulse or a properly designed prototype pulse, well-localized in both time and frequency domains, and we show that such a technique can be applied to our discrete-time blockbased model. We highlight the similarities and the differences between some recently proposed multi-carrier modulations based on DCT and DST to mathematical concepts such as the Wilson base, that in turn will be used to develop a novel and effective multi-carrier format, ready to be employed in practical communication systems. All analyzed modulations will be compared in terms of spectral efficiency and BER performance in practical implementations.
\end{abstract}

\section{INTRODUCTION}

Orthogonal Frequency-Division Multiplexing (OFDM) is an efficient modulation technique belonging to the wide class of multi-carrier modulations, which is particularly suited for transmissions over linear frequency-selective channels. It is in fact well known that, by means of OFDM, a linear time-invariant (LTI) channel can be decomposed into a set of orthogonal, interference-free sub-channels [1], and exact maximum a posteriori (MAP) detection can be carried out on a symbol-by-symbol basis, thus without the need for complex equalization techniques. Other advantages of OFDM include, among others, improved spectral efficiency on frequencyselective channels by means of power allocation, easier resource allocation in multi-user scenarios, and, last but not least, low-complexity implementation through the use of fast algorithms for discrete Fourier transform (DFT). As a consequence, OFDM has been already implemented in both wireline applications (such as in the digital subscriber line (DSL) standards) as well as in a wide range of wireless applications, ranging from the digital audio and video broadcasting (DABT, DVB-T, DVB-SH, DVB-H) standards, to the local and metropolitan area networks (WLANs and WMANs).

On the other hand, one of the OFDM most critical drawbacks, namely the increased sensitivity to the channel impulse response (CIR) time variations, has restricted the application of OFDM to scenarios characterized by sufficiently slow variations. In the presence of a rapidly time-varying CIR, where time selectivity stems for example from the Doppler effect or the oscillators' phase noise, the orthogonality between the sub-carriers is destroyed, inter-carrier interference (ICI) appears [2], [3], and complex equalization techniques must be employed to cope with the latter effect [4], [5].

Recently, multi-carrier modulations different from OFDM have been proposed to reduce the sensitivity to time-variations, in order to be able to effectively employ such modulations on doubly-selective channels. For example, some authors have proposed to use either the discrete cosine transform (DCT) or the discrete sine transform (DST) as an alternative to the DFT in the modulator/demodulator implementation, for which fast implementations exist (the resulting modulation formats are denoted respectively as DCT-OFDM and DST-OFDM, in order to distinguish them from the previously mentioned DFT-OFDM scheme). In particular in [6], the authors derive conditions on a guard sequence and a front-end prefilter for which a frequency selective channel can be diagonalized into orthogonal, interference-free, parallel sub-channels when DCT-OFDM is considered. Moreover, in [7] it is shown that DCT-OFDM outperforms DFT-OFDM system over AWGN channel affected by a carrier frequency offset and over a frequency-selective fast Rayleigh fading channel, while in [8] it is proved that DCT-OFDM and DST-OFDM are more robust to the interference, at the price of a dramatical decrease of the spectral efficiency due to a large overhead. Moreover, it was also pointed out that such overhead can be avoided by interspersing the two above mentioned discrete trigonometric transforms (DTT) in the modulator stage (DTT-OFDM), by transmitting the DCT coefficients on the even subcarriers and the DST coefficients on the odd subcarriers [8]. However, this approach was not pursued further due to the fact that it leads to signals no longer orthogonal on LTI channels (though orthogonality is instead ensured on non-dispersive channels). We remark that no scheme able to maintain orthogonality on a doubly-selective stochastic channel exists [9], thus orthogonality is not a realistic performance measure when doublyselective channels are considered.

An alternative approach to combat ICI, recently pursued by several authors and denoted as pulseshape OFDM (see, for example, [10]-[13] and references therein), consists in a suitable design of the prototype pulse, so as to obtain a better time-frequency localization with respect to the rectangular pulse employed in standard OFDM, and thus reduce ICI. These approaches, that can be applied to any of the above mentioned multi-carrier formats, allow low-complexity symbol-bysymbol detection, even on rapidly time-varying channels.

The aim of this paper is threefold. Firstly, we propose 


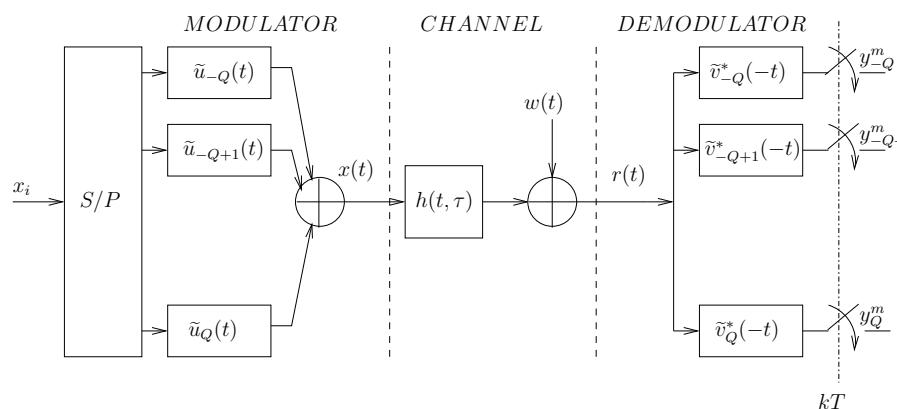

Fig. 1. Ideal continuous-time filter-bank system.

discrete-time transmitter and receiver models aiming at the practical implementation of various multi-carrier modulation formats in realistic communication systems. Secondly, we show that the performance of the above mentioned recently proposed multi-carrier techniques, namely DCT-OFDM and DTT-OFDM [8], can be easily outperformed with a suitable pulse design. Finally, inspired by the Wilson base [14, Section 4.2], that turns out to be a clever way to design welllocalized orthogonal Gabor frames with high time-frequency efficiency that avoid the limiting factor due to the BalianLow theorem [14], [15], we derive a practical multi-carrier modulation with excellent performance on doubly-selective channels. For all the above mentioned modulation schemes, we first assume a rectangular prototype pulse, as is usually done in the literature [1], [8], then we design a suitable prototype pulse, well-localized in both the time and frequency domains, by means of the technique proposed in [16]. The bit-errorrate (BER) performance of all the considered schemes, when a symbol-by-symbol detection strategy is employed, will be compared on doubly-selective channels of practical interest, as the indoor terrestrial wireless channel with mobile terminals.

\section{System Model}

We consider a linear modulation scheme as shown in Fig. 1, in which the information symbols $\left\{x_{i}\right\}$, generated by a source at a rate $R=(2 Q+1) / T$, are serial-to-parallel converted to obtain length- $(2 Q+1)$ blocks (also known as OFDM blocks), $Q$ being an integer design parameter. We will use the following notation to denote the symbols:

$$
x_{n}^{m}=x_{m(2 Q+1)+n} \quad \text { with } \quad \begin{aligned}
& n=-Q,-Q+1, \ldots, Q \\
& m \in \mathbb{Z}
\end{aligned}
$$

where $m$ represents the block index (also denoted as time index), $n$ is the index identifying a symbol position inside a block, and $T$ is the block period. The transmitted symbols $\left\{x_{i}\right\}$ are zero-mean independent random variables (r.v.s) belonging to a given complex constellation. The transmitted signal, which is obtained by modulating a set of continuous-time filters $\left\{\widetilde{u}_{n}(t)\right\}$, reads

$$
x(t)=\sum_{m \in \mathbb{Z}} \sum_{n=-Q}^{Q} x_{n}^{m} \widetilde{u}_{n}(t-m T) .
$$

Filters $\left\{\widetilde{u}_{n}(t)\right\}$ will be chosen equal to a base pulse (either a rectangular pulse or an appropriately designed pulse, according to the approach described in [12]) with a suitable multiplexing in the frequency domain, as in the uniform filter-bank approach [17, Chapter 9]. Hence, index $n$ is usually referred to as the frequency (or subcarrier) index. We will define the spectral efficiency $\eta$, expressed in symb/s/Hz, as the amount of code symbols that can be loaded on a time-frequency region characterized by a unitary bandwidth and timewidth. All the considered modulation formats will be designed so that they achieve the same spectral effiency, in order to carry out a fair comparison.

After transmission over a noisy doubly-selective channel, the received signal $r(t)$ is

$$
r(t)=\int_{-\infty}^{+\infty} h(t, \tau) x(t-\tau) d \tau+w(t)
$$

where the zero-mean Gaussian random process $h(t, \tau)$ denotes the time-varying channel impulse response (CIR). A widesense stationary uncorrelated scattering (WSSUS) channel is assumed, and $h(t, \tau)=0$ if $\tau<0$ or $\tau>\tau_{\max }$, being $\tau_{\max }$ the maximal excess delay of the channel. $\tau_{\max }$ gives an indication about the channel spreading in the time domain. A similar parameter, denoted as $\nu_{\max }$ (maximal Doppler spread), quantifies the channel spreading in the frequency domain [18]. $w(t)$ is a complex white Gaussian process with power spectral density $2 N_{0}$.

At the receiver, the signal is processed by a bank of $2 Q+1$ filters. The $r$-th sample at the output of the $k$-th filter $\left\{\widetilde{v}_{k}^{*}(t)\right\}$ is

$$
y_{k}^{r} \triangleq y_{k}(r T)=\int_{-\infty}^{+\infty} r(\tau) \widetilde{v}_{k}^{*}(\tau-r T), k=-Q, \ldots, Q .
$$

In order to obtain a practical implementation model for the mentioned filter-bank scheme, we resort to a discrete-time model. In particular each impulse response filter $\widetilde{u}_{n}(t)$ (and similarly for $\left.\widetilde{v}_{k}(t)\right)$ is approximately represented by

$$
\widetilde{u}_{n}(t) \simeq \sum_{i \in \mathbb{Z}} \widetilde{u}_{n}(i) \sqcap\left(\frac{t}{T_{s}}-i\right)
$$

where $\widetilde{u}_{n}(i) \triangleq \widetilde{u}_{n}\left(i T_{s}\right), T_{s} \triangleq T / N$ ( $N$ is the oversampling factor) and $\sqcap(t)$ is a rectangular pulse defined as

$$
\sqcap(\alpha) \triangleq \begin{cases}1 & \text { if } 0 \leq \alpha \leq 1 \\ 0 & \text { otherwise }\end{cases}
$$

The discrete-time representations of the filters, according to (4), is sufficiently accurate provided that a large enough oversampling factor $N$ is employed. Under this assumption, an equivalent discrete-time representation of the transceiver is shown in Fig. 2, where the two square filters $\sqcap(\cdot)$ play the role of digital-to-analog and analog-to-digital converters, respectively.

The discrete-time representation of the transmitted signal (1) is

$$
x(t)=\sqrt{\frac{1}{T_{s}}} \sum_{m \in \mathbb{Z}} \sum_{n=-Q}^{Q} x_{n}^{m} \sum_{i} \widetilde{u}_{n}(i) \sqcap\left(\frac{t-m T}{T_{s}}-i\right)
$$




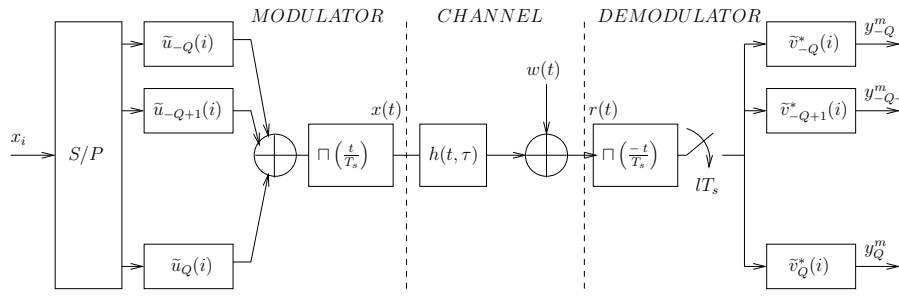

Fig. 2. Practical oversampled discrete-time filter-bank system

where the constant $\sqrt{1 / T_{s}}$ is an energy normalization term. The discrete-time received samples can be obtained by using (6) and (2) in (3), thus obtaining

$$
\begin{aligned}
y_{k}^{r} & =\sqrt{\frac{1}{T_{s}}} \sum_{l \in \mathbb{Z}} \widetilde{v}_{k}^{*}(l) \int_{-\infty}^{\infty} r(t) \sqcap\left(\frac{t-r T}{T_{s}}-l\right) d t \\
& =\sum_{m \in \mathbb{Z}} \sum_{n=-Q}^{Q} H_{k, n}^{r, m} x_{n}^{m}+n_{k}^{r} \text { with } \begin{array}{l}
k=-Q, \ldots, Q_{(7)} \\
r \in \mathbb{N}
\end{array}
\end{aligned}
$$

where

$$
H_{k, n}^{r, m} \triangleq \sum_{l \in \mathbb{Z}} \widetilde{v}_{k}^{*}(l-r N) \sum_{i \in \mathbb{Z}} \widetilde{u}_{n}(i-m N) \widetilde{h}_{l, i}
$$

with

$$
\widetilde{h}_{l, i} \triangleq \frac{1}{T_{s}} \iint_{-\infty}^{\infty} h(t, \tau) \sqcap\left(\frac{t-\tau}{T_{s}}-i\right) \sqcap\left(\frac{t}{T_{s}}-l\right) d \tau d t
$$

and

$$
n_{k}^{r} \triangleq \sqrt{\frac{1}{T_{s}}} \sum_{l \in \mathbb{Z}} \widetilde{v}_{k}^{*}(l-r N) \int_{-\infty}^{\infty} w(t) \sqcap\left(\frac{t}{T_{s}}-l\right) d t .
$$

From (9), the discrete-time CIR $\widetilde{h}_{l, i}$ has support over $0 \leq i \leq$ $L$, where $L=\left\lfloor\tau_{\max } / T_{s}\right\rfloor+1(\lfloor t\rfloor$ means round $t$ down to the nearest integer).

Eq. (7) shows that, unless $H_{k, n}^{r, m}=\delta_{n-k} \delta_{r-m}$ with probability one (being $\delta_{\ell}$ the Kronecker delta), interference among the various received samples arises, both in the time-domain and in the frequency-domain. Hence, a detection scheme based on the MAP criterion would in principle involve a complex processing to cope with such interference. Moreover, the discrete-time noise samples $n_{k}^{r}$ can be correlated, thus further complicating the development of MAP receivers. In order to keep the computational complexity of the receiver low, filter banks $\left\{\tilde{u}_{n}\right\}_{n}$ and $\left\{\tilde{v}_{n}\right\}_{n}$ can be designed with the aim of ensuring that $H_{k, n}^{r, m}$ is small, for example in a mean square error (MSE) sense [19], when $k \neq n$ or $r \neq m$. In this way, a low-complexity symbol-by-symbol receiver can be used without incurring a large performance penalties. In the following, a symbol-by-symbol receiver will always be assumed, although we point out that more involved equalization algorithms, for example based on the soft-interference cancellation (SIC) principle [20], could be employed.

\section{MulticARRIER Systems}

We now show four different approaches to design transmitter and receiver filters. To the best of the authors' knowledge, the last one has never been proposed for practical systems in the literature. We remark that all methods consist in a suitable multiplexing in the frequency domain of a prototype pulse: although rectangular pulses are commonly employed (e.g., the use of rectangular pulses have been proposed for DCTOFDM [8]), their slow frequency-domain decay behaviour is responsible for their poor performance on doubly-selective channels, due to the strong interference they experience [2]. Hence, more localized pulses in the time-frequency domain have been recently proposed [10]-[13]. In this paper, we will consider the pulse stemming from the method proposed in [12].

\section{A. DFT-OFDM}

We now derive the first modulation scheme based on DFT implementation. In this case, the frequency response of the filters is obtained by shifting the frequency response of a prototype filter by $F_{n}=n F$. In particular, denoting as $u(i)$ the oversampled version of the prototype filter $u(t)$ at $t_{i}=i T_{s}=i T / N$, we find

$$
\widetilde{u}_{n}(i)=\widetilde{v}_{n}(i) \triangleq u(i) e^{j 2 \pi F_{n} t_{i}}=u(i) e^{j 2 \pi \frac{n i}{N} F T} .
$$

Since $T$ is the OFDM symbol period and $F$ the carrier separation, each coded symbols $x_{n}^{m}$ can be associated to the point $\left(T_{m}, F_{n}\right)=(m T, n F)$ of a bidimensional grid in the time-frequency plane [12]. Hence, defining $\rho \triangleq F T$, the inverse of $\rho$ can be seen as a measure of the spectral efficiency $\eta$ (in terms of data symbols per seconds per Hertz), since higher $\rho^{-1}$ values lead to reduce space-frequency distance between symbols (i.e., reduced distance between two adjacent grid points). In order to derive modulation schemes for which a fast implementation exists, we just consider rational $\rho$ values, hence $\rho=q / p$ where $q$ and $p$ are integers and $q \geq p$. From the above assumption (11) becomes

$$
\widetilde{u}_{n}(i)=\widetilde{v}_{n}(i)=u(i) e^{j 2 \pi \frac{n i q}{N p}} .
$$

Thus, replacing (11) in (6) and in (7), we derive that, for each transmitted OFDM block $\left\{x_{n}^{m}\right\}_{n}$, the modulator has to compute an $N p$-IDFT (inverse DFT) while the demodulator must perform an $N p$-DFT. From that, it is clear that when $p=1$, as in the case of $\rho=1$, we have a minimum in the (I)DFT dimension (which is $N$ ), while in all other cases (i.e, when $\rho$ is not integer) the (I)DFT length is increased to $N p$. For all the following multi-carrier schemes the same considerations hold true, where the DFT is replaced in turn by DCT or DST or both.

For all employed modulation schemes, we will consider a rectangular prototype length- $T$ pulse to obtain DMT (Discrete Multitone) schemes, and a suitable optimized pulse well localized in both time and frequency domains [12] to achieve PS (pulseshape) schemes. Although there is no scheme able to diagonalize a doubly-selective stochastic channel into orthogonal, interference-free, subchannels, when the channel is LTI a method to completely remove interference can be reached exploiting a length- $L$ cyclic prefix $(\mathrm{CP})$ in the DFTDMT scheme. In details we fix a symbol period $T=N^{\prime} T_{s}$ where $N^{\prime}=N+L$ and $F=1 /\left(N T_{s}\right)$. Moreover the prototype pulse has support $T$ at the transmitter and $T-L T_{s}$ at the receiver. In other words, after each IDFT the $L$ final 
symbols are repeated at the beginnig of the block and are then discarded before the DFT at the receiver. In conclusion LTI channel orthogonalization can be achieved by an energy loss due to the $L$ symbols of the $\mathrm{CP}$, and with a spectral efficiency loss due to the increase of $\rho$ from 1 to $1+L / N$.

\section{B. DCT-OFDM}

In the modulation scheme employing DCT as an alternative to DFT [8], transmitter and receiver filters are obtained by modulating the prototype filter $u(t)$ by cosine functions. Sampling at $t_{i}=(i+1 / 2) T_{s}$, we find

$\widetilde{u}_{n}(i)=\widetilde{v}_{n}(i)=u(i) \sqrt{\beta_{n}} \cos \left[\pi F_{n} t_{i}\right]=u(i) \sqrt{N p} C_{N p}(n q, i)$

where we denoted by $C_{N}(n, i)$ the basis function of DCTII [21]:

$$
C_{N}(n, i) \triangleq \sqrt{\frac{\beta_{n}}{N}} \cos \left[\pi \frac{n(i+1 / 2)}{N}\right]
$$

and $\beta_{n}$ is 1 if $n=0$ and 2 if $n=1, \ldots, N-1$. Hence, when $\rho=q / p$, for each OFDM block the transmitter and the receiver carry out an $N p$-IDCT and an $N p$-DCT, respectively. Both the rectangular and the optimized pulse $u(i)$ can be employed and, as in the case of DFT-DMT, DCT-DMT can also reach perfect channel orthogonalization over LTI channels. However, in this case not only a CP (as described in Sect. III-A) is necessary, but also a symmetric extension at the output of each $N p$-IDCT must be employed [8]. Since it can be easily verified that a $N / 2$-IDCT followed by a symmetric extension is equivalent to a $N$-IDCT whose odd inputs are set to zero, the information throughput is reduced of a factor $1 / 2$ with respect to the DMTDFT scheme and $\rho=2(1+L / N)$. We will denote the scheme exploiting rectangular pulse and symmetric extension as DCTMAN.

\section{DTT-OFDM}

In [8], Mandyam also proposed a scheme based on both DCT and DST, with a rectangular prototype pulse. In details, the prototype filter $u(t)$ is modulated by a cosine function for the even subcarriers and by a sine function for the odd sucarriers. By sampling $\widetilde{u}_{n}(t)$ and $\widetilde{v}_{n}(t)$ at $t_{i}=(i+1 / 2) T_{s}$, we obtain

$$
\widetilde{u}_{n}(i)= \begin{cases}u(i) \sqrt{N p} C_{N p}(n q, i) & \text { if } n \text { even } \\ u(i) \sqrt{N p} S_{N p}(n q+q-1, i) & \text { if } \quad n \text { odd }\end{cases}
$$

and the same is for $\widetilde{v}_{k}(i)$, where we have defined the basis function of DST-II [21]:

$$
S_{N}(n, i) \triangleq \sqrt{\frac{\gamma_{n}}{N}} \sin \left[\pi \frac{(n+1)(i+1 / 2)}{N}\right]
$$

and $\gamma_{n}$ is 1 if $n=N-1$ and 2 if $n=0, \ldots, N-2$. Although in [8] this scheme is not further analyzed since it cannot lead to a complete interference cancellation over LTI channels, this is not a significant drawback over doubly-selective channels [10].

\section{ODTT-OFDM}

In order to design well-localized orthogonal Gabor frames, Wilson proposed a continuous-time base [14, Section 4.2] where subcarriers are modulated by cosine and sine functions (as in the PS-DTT case). In particular, the base functions $\widetilde{u}_{n}(t-m T)$ are described by

$$
\widetilde{u}_{2 \ell+\sigma}(t-m T)= \begin{cases}G_{0, m}(t) & \ell=0 \text { and } \sigma=1 \\ G_{\ell, 2 m+\sigma}(t) & \ell>0\end{cases}
$$

where $\sigma \in\{0,1\}, \ell \in \mathbb{N} \backslash\{\ell=0, \sigma=0\}$ and $G_{n, m}(t)$ is defined as

$$
\begin{cases}u(t-m T) & n=0 \\ \sqrt{2} u(t-m T / 2) \cos (2 \pi n F t) & n>0 \text { and } n+m \text { even } \\ \sqrt{2} u(t-m T / 2) \sin (2 \pi n F t) & n>0 \text { and } n+m \text { odd. }\end{cases}
$$

Following these lines, with respect to the the PS-DTT case we introduce a new multi-carrier discrete-time system where half filters are time shifted of half a symbol period $T$. We consider rational values of $\rho(\rho=q / p)$ and by sampling $\widetilde{u}_{n}(t)$ at $t_{i}=(i+1 / 2) T_{s}$, we derive the following expression for $\widetilde{u}_{n}(i)$ (and the same is for $\widetilde{v}_{n}(i)$ )

$$
\begin{cases}\sqrt{N p} u(i) C_{N p}(0, i) & n=1 \\ \sqrt{N p} u(i) C_{N p}(n q, i) & {[n]_{4}=0 \text { and } n>1} \\ \sqrt{N p} u(i-N / 2) S_{N p}(n q-q-1, i) & {[n]_{4}=1 \text { and } n>1} \\ \sqrt{N p} u(i) S_{N p}(n q-1, i) & {[n]_{4}=2} \\ \sqrt{N p} u(i-N / 2) C_{N p}(n q-q, i) & {[n]_{4}=3}\end{cases}
$$

where $n$ spans form 1 to $2 Q+1$. We will denote this scheme as ODTT-OFDM (Offset-DTT).

\section{Simulation Results}

We will compare all the described schemes over doublyselective channels. As a measure of the impairment produced by the channel we consider the so-called spread factor $\lambda$ defined as $\lambda=\tau_{\max } \nu_{\max }$. In order to get a fair comparison, we will consider the same spectral efficiency $\rho^{-1}$, the same signal bandwidth and the same channel spread factor $\lambda$ for all considered multi-carrier systems. We expect that the PS-ODTT scheme exhibits the largest robustness to the interference generated by a doubly-selective channel, thanks to the good time-frequency localization assured by the Wilson base.

\section{CONCLUSIONS}

We have compared four multi-carrier transmissions systems in a discrete-time, oversampled domain, over doubly-selective channels. We have shown that all schemes can be implemented by resorting to fast transforms (like DFT, DCT or DST), and for all schemes we have assumed either a rectangular prototype pulse or a suitable designed base pulse well localized in both time and frequency domains. Finally, we can state that, since the ODTT scheme we have derived is inspired by the Wilson base (which is a clever way to design well-localized Gabor frames), such system is the most robust to the interference introduced by a doubly-selective channel. 


\section{REFERENCES}

[1] J. A. C. Bingham, "Multicarrier modulation for data transmission: An idea whose time has come," IEEE Commun. Mag., pp. 5-14, May 1990.

[2] X. Cai and G. B. Giannakis, "Bounding performance and suppressing intercarrier interference in wireless mobile OFDM," IEEE Trans. Commun., vol. 51, pp. 2047-2056, Dec. 2003.

[3] L. Tomba, "On the effect of Wiener phase noise in OFDM systems," IEEE Trans. Commun., vol. 46, pp. 580-583, May 1998.

[4] A. Stamoulis, S. N. Diggavi, and N. Al-Dhahir, "Intercarrier interference in MIMO OFDM," IEEE Trans. Signal Processing, vol. 50, pp. 24512464, Oct. 2002

[5] S. Hwang and P. Schniter, "Efficient communication over highly spread underwater acoustic channels," in Proc. of the ACM Intern. Workshop on UnderWater Networks (WUWNet), Sept. 2007.

[6] N. Al-Dhahir, H. Minn, and S. Satish, "Optimum DCT-based multicarrier transceivers for frequency-selective channels," IEEE Trans. on Communications, vol. 54, pp. 911-921, May 2006.

[7] P. Tan and N. C. Beaulieu, "A comparison of DCT-based OFDM and DFT-based OFDM in frequency offset and fading channels," IEEE Trans. Commun., vol. 54, pp. 2113-2125, Nov. 2006.

[8] G. D. Mandyam, "Sinusoidal transforms in OFDM systems," IEEE Trans. on Broadcasting, vol. 50, pp. 172-184, June 2004.

[9] W. Kozek and A. F. Molisch, "On the eigenstructure of underspread WSSUS channels," in Proc. of the IEEE Workshop on Signal Proc. Adv. in Wireless Comm. (SPAWC), (Paris, France), pp. 325-328, Apr. 1997.

[10] W. Kozek and A. F. Molisch, "Nonorthogonal pulseshapes for multicarrier communications in doubly dispersive channels," IEEE J. Select. Areas Commun., vol. 16, pp. 1579-1589, Oct. 1998.
[11] G. Matz and F. Hlawatsch, "Time-varying communication channels: fundamentals, recent developments, and open problems," in Proc. European Signal Processing Conference (EUSIPCO), (Florence, Italy), Sept. 2006.

[12] T. Strohmer and S. Beaver, "Optimal OFDM design for time-frequency dispersive channels," IEEE Trans. Commun., vol. 51, pp. 1111-1122, July 2003.

[13] A. Vahlin and N. Holte, "Optimal finite duration pulses for OFDM," IEEE Trans. Commun., vol. 44, pp. 10-14, Jan. 1996.

[14] I. Daubechies, Ten Lectures on Wavelets. SIAM J., 1991.

[15] R. L. Allen and D. W. Mills, Signal Analysis-time, frequency, scale, and structure. John Wiley \& Sons, 2004.

[16] B. L. Floch, M. Alard, and C. Berrou, "Coded orthogonal frequency division multiplex," Proc. IEEE, vol. 83, pp. 982-996, June 1995.

[17] N. Benvenuto and G. Cherubini, Algorithms for Communications Systems and Their Applications. John Wiley \& Sons, 2002.

[18] P. A. Bello, "Characterization of randomly time-variant linear channels," IEEE Trans. Commun. Systems, vol. 11, pp. 360-393, 1963.

[19] A. Barbieri, G. Caire, and U. Mitra, "An approximate eigenmode decomposition for doubly-selective wireless channels," in Proc. ICASSP, (Las Vegas, NV), Mar.-Apr. 2008.

[20] J. Boutros and G. Caire, "Iterative multiuser joint decoding: unified framework and asymptotic analysis," IEEE Trans. Inform. Theory, vol. 48, pp. 1772-1793, July 2002.

[21] S. Martucci, "Symmetric convolution and the discrete sine and cosine transforms," IEEE Trans. on Signal Processing, vol. 42, pp. 1038-1051, May 1994. 